\title{
Carbon Composites ist Mitglied von "go-cluster"
}

Der Carbon Composites (CCeV) ist Mitte November in das bundesweite Projekt "go-cluster: Exzellent vernetzt" aufgenommen worden. Das Projekt "go-cluster" wurde im Juli 2012 als Nachfolger der Initiative „Kompetenznetze Deutschland" eingeführt. Gefördert wird das Projekt vom Bundesministerium für Wirtschaft und Technologie (BMWi), mit der Umsetzung des Projektes ist die VDINDE-IT beauftragt. Projektziele sind unter anderem die Weiterentwicklung leistungsfähiger Innovationscluster in Deutschland, die Analyse internationaler Clusterpolitik sowie die Förderung der Umsetzung neuartiger Clusterservices. Damit soll "gocluster" für ein verbessertes Clustermanage- ment, für neuartige Servicekonzepte, die internationale Sichtbarkeit der Cluster und eine aktiv gestaltete Clusterpolitik in Deutschland und Europa stehen.

Carbon Composites e.V. (CCeV) ist nach eigenen Angaben ein Verbund von Unternehmen und Forschungseinrichtungen, der die gesamte Wertschöpfungskette der HochleistungsFaserverbundwerkstoffe abdeckt. „CCeV vernetzt Forschung und Wirtschaft in Deutschland, Österreich und der Schweiz", heißt es.

Der Verein versteht sich als Kompetenznetzwerk zur Förderung der Anwendung von Faserverbundwerkstoffen. Die Aktivitäten des CCeV sind auf die Produktgruppe
"Marktfähige Hochleistungs-Faserverbundstrukturen" ausgerichtet. Schwerpunkte liegen auf Faserverbundstrukturen mit Kunststoffmatrices, wie sie aus vielen Anwendungen auch einer breiteren Öffentlichkeit bekannt sind, sowie auf Faserverbundstrukturen mit Keramikmatrices mit ihren höheren Temperatur- und Verschleißbeständigkeiten.

Der CCeV wurde 2007 gegründet und umfasst aktuell 176 Mitglieder, darunter 35 Forschungseinrichtungen, 41 Großunternehmen, 88 kleine und mittlere Unternehmen, fünf assoziierte Mitglieder sowie sieben unterstützende Organisationen. Sitz des Vereins ist Augsburg.
Der Markt für CFK soll in den kommenden Jahren um 13 bis $17 \%$ wachsen. Dies berichtete der Geschäftsführer des Carbon Composites e.V. (CCeV), Dr. Hans-Wolfgang Schröder auf der AVK-Jahrestagung in Düsseldorf. Der Bedarf an Carbonfasern (CF) lag laut Marktbericht "Der globale CFKMarkt" im Jahr 2011 bei 37.000 t und übertraf damit geringfügig die Prognosen aus dem Jahr 2010. Für das Jahr 2012 wird ein Bedarf von rund 42.000 t prognostiziert, für das Jahr 2014 sogar ein Bedarf von circa 73.000 t. Diese Prognosen würden durch die Absichtserklärungen zahlreicher Composite-Hersteller untermauert. Wie es hieß, finden sich rund 29\% der Kapazitäten zur Carbonfaserproduktion in den USA. Europa und Japan repräsentieren zusammen $50 \%$ der Weltkapazität zur CF-Herstellung, China hat einen Anteil von rund $8 \%$ - mit steigender Tendenz. Der größte Anteil mit $23 \%$ an CFK wird laut Studie für Rotorblätter in Windkraftanlagen verwendet. Im Segment Luft- und Raumfahrt (zusammen $16 \%$ ) dominiere der Flugzeugbau mit rund $11 \%$. Im Automobilbau erscheine der Einsatz von CFK mit 6 \% relativ niedrig, heißt es in der Markteinschätzung des CCeV. Mit dem geplantem Markteintritt von BMW i und der fortschreitenden Möglichkeit, CFK-Bauteile in Serie zu fertigen, seien die Wachstumsraten in diesem Bereich aber weitaus höher als in anderen Branchen.
„Analysten schätzen den CFK-Markt als solide und vor allem als einen Markt mit hohem Wachstumspotenzial ein“, betont Bernhard Jahn, Autor des Berichts. Man gehe von mindestens $13 \%$ jährlichem Wachstum aus, das zum einen auf steigende Ordereingänge bei der Luftfahrtindustrie, zum anderen auf die Automobilindustrie als einen der größten Treiber des CFK-Marktes zurückzuführen sei. Ausgehend von einem Verbrauch von rund 2000 t CFK für 2010 würden konservative Analysten hier mit einer Steigerung von 15 \% jährlich rechnen. Je nach dem Erfolg der angekündigten Elektrofahrzeuge könne es ab 2013 aber auch zu deutlich höheren Steigerungsraten kommen.

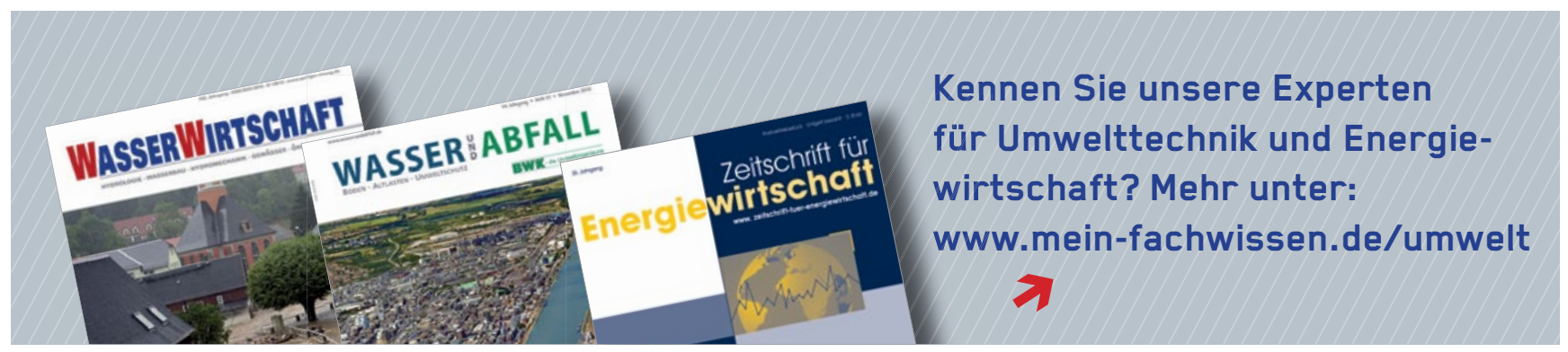

www.lightweight-design.de 